\title{
New Deduced Results in Subatomic Physics
}

\section{Bo Lehnert}

Alfvén Laboratory, Royal Institute of Technology, Stockholm, Sweden

Email: bo.lehnert@ee.kth.se

How to cite this paper: Lehnert, B. (2019) New Deduced Results in Subatomic Physics. Journal of Modern Physics, 10, 663-672.

https://doi.org/10.4236/jmp.2019.106047

Received: April 2, 2019

Accepted: May 24, 2019

Published: May 27, 2019

Copyright (c) 2019 by author(s) and Scientific Research Publishing Inc.

This work is licensed under the Creative Commons Attribution International License (CC BY 4.0).

http://creativecommons.org/licenses/by/4.0/

\section{Open Access}

\begin{abstract}
The Standard Model of elementary particles, with its associated concept of a vacuum state of empty space, leads to strongly restricted results in subatomic physics. Examples are given by vanishing rest masses and an associated spinless state of the photon. In a revised quantum electrodynamic theory by the author, new results have been deduced which cannot be obtained from the Standard Model. These are due to a vacuum state populated by Zero Point Energy and a corresponding nonzero electric charge density. This leads to a screw-shaped photon configuration with rest mass, spin and possibilities of needle radiation, to a deduced value of the elementary charge of the electron, muon and tauon being close to its experimental value, to a deduced mass being nearly equal to $125 \mathrm{GeV}$ of the Higgs particle detected at CERN, and to the discovery of large intrinsic charges of both polarities within the volume of a particle. In their turn, these charges give rise to effects of the same magnitude as that of the strong force, and can account for the binding energy of $8 \mathrm{MeV}$ of the neutron and proton. This makes a unification possible of electrodynamics with the strong nuclear force.
\end{abstract}

\section{Keywords}

Quantum Electrodynamics, Standard Model and Beyond, Zero Point Energy, Higgs Particle, Unification of Electrodynamics and the Strong Force

\section{Introduction}

The fundamental properties of elementary particles have earlier been discussed by the author in terms of subatomic physics, as given by a revised quantum electrodynamic theory $[1,2]$. These properties will here be reconsidered with special emphasis on the concepts of rest mass, spin, elementary electric charge, intrinsic electric charges, needle radiation, the strong force, the binding energy of neutrons and protons, the Higgs mass, and the relation between relativity and quantum mechanics.

The shortcomings of conventional theory, based on a vacuum state 
of empty space, will first be elucidated in Section 2. This is followed in Section 3 by a description of the revised subatomic field equations of a vacuum state populated by Zero Point Energy (ZPE). In its turn, this leads to new subatomic properties of the elementary particles, as being discussed in Section 4 .

\section{Shortcomings of Conventional Theory}

In conventional electromagnetic theory and such applications as plasma physics, particles of given electric charge and mass form the basis of corresponding macroscopic field equations. The latter are then related to a vacuum state of empty space. This theory is well established and will not be further discussed here.

A different situation arises, however, when attempts are made to treat the interior of elementary particles in terms of subatomic theory. In this section some important examples are given on what happens if the vacuum concept of empty space is being retained. It implies that the electric charge density represented by the local value $\operatorname{div} \mathbf{E}$ of the electric field strength $\mathbf{E}$ vanishes identically.

The first example concerns the conventional equations being applied to a model of the propagating photon, treated with $\operatorname{div} \mathbf{E}=0$ by means of the Hertz vector, as given by Stratton [3]. According to Chapter 9 of [1], these equations have solutions in plane, cylindrical, and spherical geometry which all lead to a vanishing total angular momentum (spin). In particular for cylindrical geometry the local spin contributions $s_{z 1}$ and $s_{z 2}$ of the TM and TE wave types become

$$
s_{z 1}=s_{z 2} \propto K^{2} n\left[J_{n}(K r)\right]^{2}(\sin 2 n \varphi)
$$

where

$$
K^{2}=(\omega / c)^{2}-k^{2}
$$

for a wave form $f(r) \exp [i(-\omega t+k z+n \varphi)]$ in a cylindrical frame $(r, \varphi, z)$ with $J_{n}(K r)$ as a Bessel function. The total integrated contribution to the spin then vanishes for all $K$ and $n$. Consequently, conventional theory does not become reconcilable with a physically relevant photon model which should possess spin according to experiments.

The second example concerns the steady state with both $\operatorname{div} \mathbf{E}=0$ and $\partial / \partial t=0$. Then the charge density $\varepsilon_{0} \operatorname{div} \mathbf{E}$ and curl $\mathbf{B}$ of the magnetic field $\mathbf{B}$ will vanish. This implies that there are no local sources of the electromagnetic field. As also being pointed out in Section 6.1 of [1], this is in agreement with Quigg [4] who states that the symmetry of the conventional field equations does not permit masses for quarks and leptons. Consequently, the existence of particle-like states rests on a nonzero div $\mathbf{E}$ and an associated broken symmetry between $\mathbf{E}$ and $\mathbf{B}$.

A third point concerns the conventional description of the photon in terms of plane waves. This is not only in contradiction with a nonzero spin, but also with the experimentally proved needle-like behavior, as described in Chapter 4.1 of [1]. Both these circumstances influence the way in which the concept of special relativity has to be transferred from a plane-wave model to a multidimensional model, such as that of cylindrical geometry later treated in this paper. Needless to say, this does not affect the general lines of thought of special relativity by Einstein. 


\section{Revised Subatomic Field Equations}

The shortcomings of conventional theory impose restrictions on a treatment of subatomic physics. A way out of this dilemma is provided by the following sections. The present treatment thus includes terms with a nonzero div $\mathbf{E}$ which are not present in a conventional analysis, and where div $\mathbf{E}$ acts somewhat like a hidden variable.

\subsection{The Zero Point Energy}

In a study of the quantum mechanical energy levels of the harmonic oscillator, M. Planck found that there is a nonzero ground level, the Zero Point Energy (ZPE), as further described in a review by Schiff [5]. The related electromagnetic vacuum fluctuations were investigated by Casimir [6] who predicted that two metal plates separated by a small spacing will attract each other, due to the fact that only small wavelengths can exist within the spacing. Such a net differential force was first demonstrated experimentally by Lamoreaux [7]. Part of the fluctuations also carry electric charge, as pointed out by Abbott [8]. Consequently, the vacuum is not merely a state of empty space, but includes photon-like modes being each of frequency $\nu$ and energy $h \nu / 2$ and which result in a real macroscopic pressure. Even if these fluctuations occur at the lowest ZPE level, their spatial inhomogeneity can produce a pressure gradient. Since the Casimir force is due to a comparatively small differential pressure, the total ZPE can become much larger. More detailed discussions are presented elsewhere [9-11].

The basis of the present subatomic field equations is thus formed by the concepts of ZPE and the Casimir force, in combination with special relativity.

\subsection{The Extended Equations}

With the basic concepts of the previous subsection, a revised quantum electrodynamic theory (RQED) is now established [1]. Thereby the new inclusion of terms with a nonzero div $\mathbf{E}$ becomes sources of electric charge and matter, in accordance with the incorporated effects of the ZPE. It thus leads to a four-dimensional representation

$$
\square A_{\mu}=\left(\frac{1}{c^{2}} \frac{\partial^{2}}{\partial t^{2}}-\nabla^{2}\right) A_{\mu}=\mu_{0} J_{\mu}
$$

of the Lorentz invariant field equations with $\mu=1,2,3,4$. Here $A_{\mu}=$ $(\mathbf{A}, i \phi / c)$ where $\mathbf{A}$ is the magnetic vector potential, $\phi$ the electrostatic potential, $c^{2}=1 / \mu_{0} \varepsilon_{0}$ where $c \cong 2.998 \times 10^{8} \mathrm{~m} / \mathrm{s}$ denotes the velocity constant of light determined by the magnetic permeability $\mu_{0}$ and the dielectric constant $\varepsilon_{0}$, and $J_{\mu}=(\mathbf{j}, i c \bar{\rho})$ where $\mathbf{j}$ stands for the electric current density and $\bar{\rho}=\varepsilon_{0} \operatorname{div} \mathbf{E}$ for the electric charge density. Since $J_{\mu}$ is relativistically invariant, it has to satisfy the condition

$$
\mathbf{j}^{2}-c^{2} \bar{\rho}^{2}=\text { const. }
$$

where the constant must vanish because $J_{\mu}$ disappears when there are no sources of charge and current density. This leads to the final form

$$
J_{\mu}=\bar{\rho}(\mathbf{C}, i c)
$$

where

$$
\mathbf{C}^{2}=c^{2}
$$


and $\mathbf{C}$ is a velocity vector with the modulus $c$.

In a three-dimensional representation the new extended subatomic field equations of the vacuum state become

$$
\begin{gathered}
c^{2} \operatorname{curl} \mathbf{B}=(\operatorname{div} \mathbf{E}) \mathbf{C}+\frac{\partial \mathbf{E}}{\partial t} \\
\operatorname{curl} \mathbf{E}=-\frac{\partial \mathbf{B}}{\partial t}
\end{gathered}
$$

where

$$
\begin{gathered}
\mathbf{B}=\operatorname{curl} \mathbf{A}, \quad \operatorname{div} \mathbf{B}=0 \\
\mathbf{E}=-\nabla \phi-\frac{\partial \mathbf{A}}{\partial t}, \quad \operatorname{div} \mathbf{E}=\bar{\rho} / \varepsilon_{0}
\end{gathered}
$$

As compared to conventional theory, these include the additional contributions from div $\mathbf{E}$ due to ZPE in Equations (7) and (10), with the vector $\mathbf{C}$ of Equation (6) replacing the velocity constant $c$. This replacement is associated with the extension of the plane-wave representation of special relativity to a two-dimensional one, such as that of cylindrical geometry with an incorporated spin concept.

\section{Resulting New Subatomic Properties}

A number of new results have been obtained from Equations (3)-(10) which cannot be deduced in terms of the Standard Model. Corresponding particle models of steady axisymmetric states thus arise from a related generating function. As shown in Chapter 6 of [1], a radially convergent such function leads to vanishing net electric charge $q_{0}$ and magnetic moment $M_{0}$, whereas a divergent one results in net values of $q_{0}$ and $M_{0}$ under certain circumstances. In this section a number of examples [2] will be reconsidered.

\subsection{Photon Model with Spin and Rest Mass}

The solution for cylindrical waves in a frame $(r, \varphi, z)$, with the velocity vector $\mathbf{C}=c(0, \cos \alpha, \sin \alpha), \cos \alpha \ll 1$, and a constant angle $\alpha$, leads to a screw-shaped configuration which includes spin and an associated very small but nonzero rest mass, as shown in Chapter 11 of [1]. The phase and group velocities become slightly smaller than $c$, the field geometry is helical with nonzero axial field components, and it becomes limited in space.

For a generating function with a convergent radial part there is an effective photon radius

$$
\hat{r}=\frac{\varepsilon \lambda_{0}}{2 \pi(\cos \alpha)}
$$

with a main wavelength $\lambda_{0}$ of a corresponding wave packet, and a parameter $\varepsilon=1$. As an illustration, $\cos \alpha \leqslant 10^{-4}$ and $\lambda_{0}=3 \times 10^{-6}$ $\mathrm{m}$ yields a photon rest mass $m_{0} \leqslant 10^{-40} \mathrm{~kg}$. The phase and group velocities then only deviate by a factor less than $5 \times 10^{-9}$ from the velocity constant $c$. The spin thus exists at the expense of a very small reduction of the momentum which is related to the axial propagation.

There is also a photon mode originating from a generating function with a divergent radial part. A revised renormalization procedure is applied to this mode, to obtain finite values for the parameters of a wave packet as shown in Chapter 11.3.5 of [1] and in [12]. The 
effective radius of Equation (11) is then obtained for $0<\varepsilon \ll 1$. With $\varepsilon \leqslant \cos \alpha \leqslant 10^{-4}$ and $\lambda_{0}=3 \times 10^{-6} \mathrm{~m}$ we then have $\hat{r} \leqslant 5 \times 10^{-7} \mathrm{~m}$. This represents a form of needle radiation.

\subsection{The Elementary Charge}

The main features of steady axisymmetric states can be deduced from the time-independent field equations of a separable generating function

$$
F(r, \theta)=G_{0} G(\rho, \theta) \quad G=R(\rho) \cdot T(\theta)
$$

in a spherical frame $(r, \theta, \varphi)$, where all relevant quantities are independent of the angle $\varphi$ and $\rho=r / r_{0}$, with $r_{0}$, as a characteristic radial dimension. To arrive at a particle with net electric charge and magnetic moment, a divergent radial part

$$
R=\rho^{-\gamma} e^{-\rho} \quad \gamma>0
$$

is chosen, in combination with a polar part $T$ of top-bottom symmetry.

The divergence problem of infinite self-energy which arises in the resulting point-charge-like geometry, can be tackled by a process of renormalization. The conventional way of proceeding is described by Ryder [13] among others, implying that the divergent behaviour is outbalanced by adding extra ad hoc counter terms to the Lagrangian. In the present approach an alternative way is chosen, where the "infinity" of the divergent system is out balanced by the "zero" of a counter-factor [14]. Such a factor is already inherent in the present theory and has not to be imposed, as shown in Chapter 7.3 of [1].

Corresponding deductions have been performed on the electron, muon, and tauon, leading to the following results:

- There is a net electric charge $q_{0}=Q_{0} f_{p}$ where $Q_{0}=\left(\varepsilon_{0} c h\right)^{1 / 2} \simeq$ $8.86 \times 10^{-19} \mathrm{C}$ and $f_{p}$ is a dimensionless profile factor. $Q_{0}$ is related to the fine-structure constant, and it can be taken as an example where relativity is linked with quantum mechanics through the present theory.

- A variational analysis on the factor $f_{p}$ finally results in a minimum elementary charge $q_{0}=1.60 \times 10^{-19} \mathrm{C}$ within an uncertainty a few percent as compared to the experimental value of $e[1,15,16]$.

- The product of the rest mass $m_{0}$ and the magnetic moment $M_{0}$ becomes

$$
m_{0} M_{0}=(c h / 4 \pi)\left[1+\left(e^{2} / 4 \pi \varepsilon_{0} c h\right)\right]=8.46 \times 10^{-34} \mathrm{~kg} \cdot \mathrm{A} \cdot \mathrm{m}^{2}
$$

This agrees with experimental data for $m_{0} M_{0}$ of the electron, muon and tauon within the limit of a few percent.

The obtained characteristic charge $Q_{0}$ and the deduced minimum value of $q_{0}$ being close to the experimental value of the elementary charge $e$, thus suggests that the latter should be considered as a deduced quantity, and not as a fundamental and independent parameter.

\subsection{The $\mathrm{Z}$ Boson and the Higgs Particle}

The treatment of the vacuum as an empty space leads to a number of shortcomings, such as to a missing rest mass of elementary particles. 
This point was made clear by P. W. Higgs and F. Englert among others. The theory by Higgs [17] is based on a spontaneous nonlinear mechanism of symmetry breaking of an empty vacuum state, by which an unstable boson of unspecified but large rest mass is being formed. This particle has zero net electric charge and zero spin. In its turn, the same particle is considered to decay into a set of all other lighter and massive elementary particles. An open point concerns the theoretically undetermined value of the rest mass.

A particle was later discovered at the projects ATLAS [18] and CMS [19] of CERN, being unstable, having vanishing net electric charge and spin, and with a rest mass of $125 \mathrm{GeV}$. This discovery was understood to be a confirmation of the heavy particle due to the theory by Higgs.

An alternative and different way of explaining the results by CERN has been presented by the author [20-22], as being characterized by intrinsic linear broken symmetry. It starts with a model of the $\mathrm{Z}$ boson determined from a convergent axially symmetric generating function. From superposition of the solutions of two such Z boson models with antiparallel spin directions, a composite model can be formed. With a mass of $91 \mathrm{GeV}$ for each of the original Bosons, the composite particle obtains characteristical data given by lacking electric charge, magnetic field, and spin. It is purely electrostatic and highly unstable, and has a minimum mass close to $125 \mathrm{GeV}$. This appears to be in good agreement with the results by CERN. The composite particle solution is also consistent with the point made by Quigg [4] that the Higgs is perhaps not a truly fundamental particle, but is built out of as yet unobserved constituents.

\subsection{Intrinsic Charges and the Strong Force}

The local nonzero behaviour of $\operatorname{div} \mathbf{E}$ does not only generate a net integrated electric elementary charge $e$, but also intrinsic integrated electric charges $\pm Q_{i}$ of both polarities within various parts of the volume of an elementary particle $[1,2,23]$. This can be demonstrated by a model of an individual quark being deduced from a convergent generating function, in a frame of spherical coordinates $(r, \theta, \varphi)$ being independent of $\varphi$. We further consider a tentative model of the neutron, given by three individual quarks which are mutually bound to each other, to form a triangular configuration [24]. In a first simplified treatment equal models are used for these quarks.

The following points concern the model of an individual quark:

- In the triangular configuration each quark is assumed to have a mass of $5.57 \times 10^{-28} \mathrm{~kg}$ being a third of the neutron mass.

- Integration of the local electric charge density over various parts of the quark volume results in large positive and negative intrinsic charges $\pm Q_{i}$ where $Q_{i} / e \cong 19.9$. Thus $Q_{i}$ becomes an order of magnitude larger than the elementary charge.

Concerning the interaction between two quarks, the following points should be observed:

- The source terms of the present theory lead to a volume force density

$$
\mathbf{f}=\bar{\rho} \mathbf{E}_{i} \quad \mathbf{E}_{i}=\mathbf{E}+\mathbf{C} \times \mathbf{B}
$$

Here part of the electric contribution $\mathbf{E}$ is outbalanced by the magnetic contribution $\mathbf{C} \times \mathbf{B}$. Therefore the total field $\mathbf{E}_{i}$ only 
becomes noticeable within the innermost part of the quark volume. The result $\left(Q_{i} / e\right)^{2}=396$ implies that the interaction due to $\mathbf{E}_{i}$ would become more than two orders of magnitude larger than that arising between two elementary charges. But in the real situation the added influence of $\mathbf{C} \times \mathbf{B}$ partly outbalances that of $\mathbf{E}$.

- The total interaction between two quarks in the triangular configuration thus is of short-range character, and becomes about two orders of magnitude larger than that between two elementary charges.

- The mutual quark force and its corresponding work can be determined in terms of the distance between the quark centra. It is obtained from the force due to the local intrinsic charge density of quark number I, in combination with the reversed total field $\mathbf{E}_{i}$ generated by quark number II.

- When gradually shrinking the distance between the quark centra, a corresponding work of the mutual forces and their associated potential distributions is being traversed. This results in an outer potential barrier, followed by an intermediate well, and thus ending at an additional barrier. The mutual quark interaction thus becomes stable and attractive.

- An application to the neutron then leads to a well depth of about $7.7 \mathrm{MeV}$, being rather close to the binding energy of 8 $\mathrm{MeV}$ obtained from experiments reported by Bethe [24]. Since the additional net charges of the proton are comparable to the elementary charge, a similar result should also hold for the proton.

- In the triangular quark model an outer radius of about $3 \times 10^{-15}$ $\mathrm{m}$ and a core radius of about $10^{-15} \mathrm{~m}$ are obtained. These values are comparable with a nuclear radius of $1.5 \times 10^{-15} \mathrm{~m}$ given by experiments.

These deduced data are impaired by some uncertainties. Nevertheless the general outcome could still hold. The obtained short-range character and the order of magnitude of the attractive and stable interaction thus gives a clear indication that it should become possible to unify the fundamental phenomena of electromagnetics and the strong nuclear force.

\subsection{Special Relativity versus Quantum Mechanics}

In quantum mechanics an instantaneous behaviour of entangled states can take place through long-range interaction. A vacuum state of empty space leads on the other hand to a limited relativistic velocity propagation in subatomic physics. When $\operatorname{div} \mathbf{E}=0$, Equations (7)(10) thus result in a conventional transverse electromagnetic EM wave, with the velocity $c$. This is not reconcilable with instantaneous longrange interaction.

A possible way out of this dilemma [25] may be provided by the transition to a vacuum state with $\mathrm{ZPE}$ and $\operatorname{div} \mathbf{E} \neq 0$. Equations (7)(10) then lead to two wave types. The first is an EMS nontransverse electromagnetic space-charge wave with $\operatorname{curl} \mathbf{E} \neq 0$ and a limited relativistic propagation determined by the velocity constant $c$. The second 
type is an $\mathrm{S}$ wave of purely longitudinal electric space-charge character with curl $\mathbf{E}=0$. This wave depends only on the dielectric constant $\varepsilon_{0}$, has no relation to relativity, and is not limited in its velocity of propagation, as defined by the vector $\mathbf{C}$ in Equation (7).

The EMS and $\mathrm{S}$ concepts are here proposed to be combined into a system which satisfies the requirements of long-range interaction. Thus, two spatially limited EMS particle models being at a long mutual distance become linked by an $\mathrm{S}$ mode which is not limited in its speed of transition. This would imply that relativity and quantum mechanics do not necessarily have to be in conflict. But it remains so far as an open question which requires more investigation. There are the remaining questions whether the $\mathrm{S}$ mode becomes a physical reality, and how the matching of the axial electric field components of the EMS and $\mathrm{S}$ modes can be realized in detail.

\section{Conclusions}

From the present revised subatomic field equations, new results have been obtained in terms of the Zero Point Energy. These results cannot be deduced from the conventional Standard Model. They concern the spin, rest mass and needle radiation of the photon, a deduction of the value of the elementary electric charge, and of the mass of the CERN particle. The latter deduction is not related to the theory by Higgs. The present theory has further debouched into the discovery of intrinsic electric charges and related forces acting within corresponding particle models. These forces give rise to a potential well depth being rather close to the binding energy of the neutron and proton. This provides a possibility of unifying electrodynamics and the strong nuclear force.

It is improbable that all these different obtained results should merely by chance happen to agree with experimentally confirmed facts.

\section{Conflicts of Interest}

The author declares no conflicts of interest regarding the publication of this paper.

\section{References}

[1] Lehnert, B. (2014) Revised Quantum Electrodynamics. In: Dvoeglazov, V.V., Ed., Contemporary Fundamental Physics, Nova Science Publishers, Inc., New York, 1-154.

[2] Lehnert, B. (2018) Impacts of Revised Quantum Electrodynamics on Fundamental Physics. Journal of Electromagnetic Analysis and Applications, 10, 106-118.

https://doi.org/10.4236/jemaa.2018.105008

[3] Stratton, J.A. (1941) Electromagnetic Theory. 1st Edition, Ch. 1.10 and 1.11, McGraw-Hill Book Comp., Inc., New York and London.

[4] Quigg, C. (2008) The Coming Revolution in Particle Physics. Scientific American, 298, 46-53. https://doi.org/10.1038/scientificamerican0208-46

[5] Schiff, L.I. (1949) Quantum Mechanics. Ch. IV, Sec.13, McGrawHill Book Comp., Inc., New York, Toronto, London. 
[6] Casimir, H.B.G. (1948) On the Attraction between Two Perfectly Conducting Plates. Proceedings of the Koninklijke Nederlandse Akademie van Wetenschappen, 51, 793-795.

[7] Lamoreaux, S.K. (1997) Demonstration of the Casimir Force in the 0.6 to $6 \mu \mathrm{m}$ Range. Physical Review Letters, 78, 5-8. https://doi.org/10.1103/PhysRevLett.78.5

[8] Abbott, L. (1988) The Mystery of the Cosmological Constant. Scientific American, 258, 106-113. https://doi.org/10.1038/scientificamerican0588-106

[9] Lehnert, B. (2014) Some Consequences of Zero Point Energy. Journal of Electromagnetic Analysis and Applications, 6, 319327. https://doi.org/10.4236/jemaa.2014.610032

[10] Lehnert, B. (2015) Zero Point Energy Effects on Quantum Electrodynamics. Journal of Modern Physics, 6, 448-452. https://doi.org/10.4236/jmp.2015.64048

[11] Lehnert, B. (2016). On the Cosmical Zero Point Energy Density. Journal of Modern Physics, 7, 1112-1119. https://doi.org/10.4236/jmp.2016.710100

[12] Lehnert, B. (2011) The Individual Photon in Two-Slit Experiments. International Review of Physics, 5, 15-18.

[13] Ryder, L.H. (1996) Quantum Field Theory. 2nd Edition, Cambridge University Press, Cambridge.

[14] Lehnert, B. (2010) Deduced Fundamental Properties of the Electron. International Review of Physics, 4, 1-6.

[15] Lehnert, B. and Scheffel, J. (2002) On the Minimum Elementary Charge of an Extended Electromagnetic Theory. Physica Scripta, 65, 200-207. https://doi.org/10.1238/Physica.Regular.065a00200

[16] Lehnert, B. and Höök, J. (2010) An Electron Model with Elementary Charge. Journal of Plasma Physics, 76, 419-428. https://doi.org/10.1017/S0022377809990638

[17] Higgs, P.W. (1966) Spontaneous Symmetry Breakdown without Massless Bosons. Physical Review, 145, 1156-1168. https://doi.org/10.1103/PhysRev.145.1156

[18] ATLAS Collaboration. Aad, G., et al. (2012) Observation of a New Particle in the Search for the Standard Model Higgs Boson with the ATLAS Detector at the LHC. Physics Letters B, 716, 1-29. https://doi.org/10.1016/j.physletb.2012.08.020

[19] CMS Collaboration. Chatrchyan, S., et al. (2012) Observation of a New Boson at a Mass of $125 \mathrm{GeV}$ with the CMS Experiment at the LHS. Physics Letters B, 716, 30-61.

https://doi.org/10.1016/j.physletb.2012.08.021

[20] Lehnert, B. (2013) Higgs-Like Particle Due to Revised Quantum Electrodynamics. Progress in Physics, 3, 31-32.

[21] Lehnert, B. (2014) Mass-Radius Relations of Z and Higgs-Like Bosons. Progress in Physics, 10, 5-7. 
[22] Lehnert, B. (2015) Minimum Mass of a Composite Boson. Journal of Modern Physics, 6, 2074-2079.

https://doi.org/10.4236/jmp.2015.614214

[23] Lehnert, B. (2017) Intrinsic Particle Charges and the Strong Force. Journal of Modern Physics, 8, 1053-1066.

https://doi.org/10.4236/jmp.2017.87067

[24] Bethe, H.A. (1947) Elementary Nuclear Theory. Ch. 2, John Wiley and Sons, Inc., New York and Chapman and Hall, Ltd., London, 118.

[25] Lehnert, B. (2018) Some Thoughts upon Long-Range Interaction and Entangled States. Journal of Electromagnetic Analysis and Applications, 10, 193-196.

https://doi.org/10.4236/jemaa.2018.1012015 